\title{
Classical Dynamics of Muonic-Electronic Helium and Heliumlike Ions: The Allowance for the Eccentricity of the Muon and Nucleus Orbits
}

\author{
Nikolay Kryukov', Eugene Oks ${ }^{2 *}$ \\ ${ }^{1}$ Universidad Nacional Autónoma de México, Av. Universidad, Ciudad Universitaria, Coyoacán, México, Mexico \\ ${ }^{2}$ Physics Department, Auburn University, Auburn, AL, USA \\ Email: *goks@physics.auburn.edu
}

How to cite this paper: Kryukov, N. and Oks, E. (2021) Classical Dynamics of Muonic-Electronic Helium and Heliumlike Ions: The Allowance for the Eccentricity of the Muon and Nucleus Orbits. Open Journal of Microphysics, 11, 27-36.

https://doi.org/10.4236/ojm.2021.113003

Received: July 7, 2021

Accepted: August 7, 2021

Published: August 10, 2021

Copyright $\odot 2021$ by author(s) and Scientific Research Publishing Inc. This work is licensed under the Creative Commons Attribution International License (CC BY 4.0).

http://creativecommons.org/licenses/by/4.0/

(c) $\underset{\mathrm{By}}{\mathrm{By}}$ Open Access

\begin{abstract}
In the previous paper by one of us (hereafter paper I), the author considered Rydberg states of the muonic-electronic helium atom or helium-like ion and used the fact that the muon motion occurs much more rapidly than the electron motion. Assuming that the muon and nucleus orbits are circular, he applied the analytical method based on separating rapid and slow subsystems. He showed that the electron moves in an effective potential that is mathematically equivalent to the potential of a satellite orbiting an oblate planet like the Earth. He also showed that the "unperturbed" elliptical orbit of the electron engages in two precessions simultaneously: the precession of the electron orbit in the plane of the orbit and the precession of the orbital plane of the electron around the axis perpendicular to the plane of the muon and nuclear orbits. The problem remained whether or not the allowance for the ellipticity of the orbit could significantly change the results. In the present paper, we address this problem: we study how the allowance for a relatively low eccentricity $\varepsilon$ of the muon and nucleus orbits affects the motion of the electron. We derive an additional, $\boldsymbol{\varepsilon}$-dependent term in the effective potential for the motion of the electron. We show analytically that in the particular case of the planar geometry (where the electron orbit is in the plane of the muon and nucleus orbits), it leads to an additional contribution to the frequency of the precession of the electron orbit. We demonstrate that this additional, $\boldsymbol{\varepsilon}$-dependent contribution to the precession frequency of the electron orbit can reach the same order of magnitude as the primary, $\varepsilon$-independent contribution to the precession frequency. Therefore, the results of our paper seem to be important not only qualitatively, but also quantitatively.
\end{abstract}

\section{Keywords}

Muonic-Electronic Helium, Muonic-Electronic Helium-Like Ions, Rydberg 
States, Role of the Eccentricity of the Muon Orbit, Precession of the Electron Orbit

\section{Introduction}

Muonic atoms and molecules have numerous applications-see, e.g., papers [1] [2] [3] [4] [5] and references therein. These were the inspiration for studying Rydberg quasimolecules $\mu Z$ e, consisting of a nucleus of charge $Z$, a muon, and an electron [6] [7] [8].

In particular, in paper [6] the author considered Rydberg states of the muonic-electronic helium atom or helium-like ion and used the fact that the muon motion occurs much more rapidly than the electron motion. Therefore, he applied the analytical method centered on separating rapid and slow subsystems. He showed that the electron moves in an effective potential that is mathematically equivalent to the potential of a satellite orbiting an oblate planet (the Earth satellite being an example).

Further, in paper [6] it was shown that the "unperturbed" elliptical orbit of the electron engages in the following two precessions simultaneously: the precession of the electron orbit in the plane of the orbit and the precession of the orbital plane of the electron around the axis of symmetry of the muonic orbit. Despite these two precessions, the elliptical orbit of the Rydberg electron does not change its shape. The fact that the area of the elliptical orbit is conserved manifests the conservation of the square of the electron angular momentum. Thus, the system has higher than geometrical symmetry: indeed, from the geometrical symmetry (which is axial) followed only the conservation of the projection of the angular momentum on the axis of symmetry. This was a counterintuitive result of general physical interest.

In paper [6] the muon and nucleus orbits were considered circular. The problem remained whether or not the allowance for the ellipticity of the orbit could significantly change the results. In the present paper, we address this problem: we consider a more general situation where the muon and nucleus orbits are elliptical. We study how the allowance for a relatively low eccentricity $\varepsilon$ of the muon and nucleus orbits affects the motion of the electron. We derive an additional, $\varepsilon$-dependent term in the effective potential for the motion of the electron. We show analytically that in the particular case of the planar geometry (where the electron orbit is in the plane of the muon and nucleus orbits), it leads to an additional contribution to the frequency of the precession of the electron orbit. We demonstrate that this additional, $\varepsilon$-dependent contribution to the precession frequency of the electron orbit can reach the same order of magnitude as the primary, $\varepsilon$-independent contribution to the precession frequency.

\section{New Results}

As in paper [6], in this study we analyze the system consisting of a muon, an 
electron and a nucleus of charge $Z$. Both leptons are in Rydberg states such that their principal quantum numbers are $n_{\mu} \gg 1$ (for the muon) and $n_{e} \gg 1$ (for the electron). The electron is much further way from the nucleus than the muondue to the large difference in the masses of the two leptons.

In this study we consider the case of low-eccentricity orbits of the muon and the nucleus. For a Coulomb potential:

$$
U=-\frac{\alpha}{r}
$$

(where $\alpha=Z e^{2}, e$ being the electron charge), the equation of the motion in the orbital plane is:

$$
\frac{p}{r}=1+\varepsilon \cos \varphi
$$

Here:

$$
p=\frac{L^{2}}{m_{r} \alpha}, \varepsilon=\sqrt{1+\frac{2 E L^{2}}{m_{r} \alpha^{2}}}
$$

where $\varepsilon$ is the eccentricity, $(r, \varphi)$ are the polar coordinates, $E$ is the energy, $L$ in the angular momentum, and $m_{r}$ is the reduced mass of the subsystem "particle-Coulomb center".

For low-eccentricity orbits ( $\varepsilon \ll 1)$, we have:

$$
r=\frac{p}{1+\varepsilon \cos \varphi} \approx p(1-\varepsilon \cos \varphi)=r_{0}(1-\varepsilon \cos \varphi)
$$

where $r_{0}$ is the radius of the circular orbit for $\mathcal{E}=0$. As the case $\mathcal{E}=0$ was analyzed in paper [6], the Rydberg electron perceives the rapid subsystem (the nucleus and the muon) as two uniformly charged rings of radii $R_{\text {nuco }}$ and $R_{\mu 0}$, where $R_{\mu 0} / R_{\text {nucl0 }}=m_{\text {nucl }} / m_{\mu} \gg 1 \quad\left(m_{\text {nucl }}\right.$ is the mass of the nucleus $)$. The effective potential for the Rydberg electron in that case was:

$$
U_{\text {eff }}^{(0)}=-\frac{(Z-1) e^{2}}{r}-\frac{e^{2}\left(R_{\mu 0}^{2}-Z R_{\text {nucl0 }}^{2}\right)}{4 r^{3}}\left(3 \cos ^{2} \theta-1\right)
$$

In our case of $\varepsilon \ll 1$, the quantities $R_{\mu}$ and $R_{\text {nucl }}$ are the following functions of time:

$$
R_{\mu}=R_{\mu 0}[1-\varepsilon \cos (\Omega t)], R_{\text {nucl }}=R_{\text {nucl } 0}[1-\varepsilon \cos (\Omega t)]
$$

where

$$
\Omega=\sqrt{\frac{Z e^{2}}{m_{\mu r}\left(R_{\mu 0}+R_{\text {nucl0 }}\right)^{3}}} \approx \sqrt{\frac{Z e^{2}}{m_{\mu r} R_{\mu 0}^{3}}}
$$

is the frequency of revolution of the muon and of the nucleus about their center of mass, and

$$
m_{\mu r}=\frac{m_{\mu} m_{\text {nucl }}}{m_{\mu}+m_{\text {nucl }}}
$$

is the reduced mass of the pair "nucleus-muon". Substituting Equation (6) into 
Equation (5), we obtain the following time-dependent "potential":

$$
\begin{aligned}
U(t) & =U_{e f f}^{(0)}+\frac{e^{2}\left(R_{\mu 0}^{2}-Z R_{\text {nucl0 }}^{2}\right)}{2 r^{3}}\left(3 \cos ^{2} \theta-1\right) \varepsilon \cos \Omega t \\
& \equiv U_{e f f}^{(0)}+W(r, \cos \theta) \cos \Omega t
\end{aligned}
$$

The term $W(r, \cos \theta) \cos \Omega t$ can now be processed by the method of effective potentials [9] [10] [11] [12] [13] with respect to the totally unperturbed Hamiltonian:

$$
H_{0}=\frac{p^{2}}{2 m_{e r}}-\frac{(Z-1) e^{2}}{r}
$$

where

$$
m_{e r}=\frac{m_{e}\left(m_{\mu}+m_{\text {nucl }}\right)}{m_{e}+m_{\mu}+m_{\text {nucl }}}
$$

is the reduced mass of the electron orbiting the nucleus-muon pair. For helium $(Z=2), m_{e r}=0.9999$, so that for any $Z, m_{e r}$ is very close to unity. Also, because $R_{\mu 0} / R_{\text {nucl0 }} \gg 1, R_{\text {nuc } 0}$ can be ignored in the potential.

We remind that the method of effective potentials [9] [10] [11] [12] [13] allows reducing a time-dependent Hamiltonian of a physical system to an effective time-independent Hamiltonian. This is the great advantage of this method.

The zeroth-order effective potential,

$$
U_{0}=\frac{1}{4 \Omega^{2}}\left[W,\left[W, H_{0}\right]\right]=\frac{9 \varepsilon^{2} e^{4} R_{\mu 0}^{4}\left(1-2 \cos ^{2} \theta+5 \cos ^{4} \theta\right)}{16 m_{e r} \Omega^{2} r^{8}}
$$

where $W$ is defined in Equation (9) and $[P, Q]$ are the Poisson brackets, is the time-independent term for the effective potential. On substituting $\Omega$ from Equation (7) in Equation (12), we get:

$$
U_{0}=\frac{9 \varepsilon^{2} m_{\mu r} e^{2} R_{\mu 0}^{4}\left(1-2 \cos ^{2} \theta+5 \cos ^{4} \theta\right)}{16 m_{e r} Z r^{8}}
$$

Therefore, the complete effective potential in this case is:

$$
\begin{aligned}
& U_{\text {eff }}=U_{\text {eff }}^{(0)}+U_{0} \\
& =-\frac{(Z-1) e^{2}}{r}-\frac{e^{2} R_{\mu 0}^{2}}{4 r^{3}}\left(3 \cos ^{2} \theta-1\right)+\frac{9 \varepsilon^{2} m_{\mu r} e^{2} R_{\mu 0}^{4}\left(1-2 \cos ^{2} \theta+5 \cos ^{4} \theta\right)}{16 m_{e r} Z r^{8}}
\end{aligned}
$$

Next, we consider the orbits of the electron in the plane of the orbits of the muon and the nucleus, i.e., $\theta=\pi / 2$. It is easy to check, by looking at the first and second derivatives of the effective potential from Equation (14) with respect to $\theta$, that this position corresponds to the stable equilibrium. In this case, the effective potential will be a Coulomb potential with two terms of the $1 / r^{n}$-perturbation (from now on we will use the atomic units $e=m_{e}=\hbar=1$, as in the overwhelming majority of theoretical papers in atomic physics):

$$
U_{\text {eff }}=-\frac{Z-1}{r}+\frac{R_{\mu 0}^{2}}{4 r^{3}}+\frac{9 \varepsilon^{2} m_{\mu r} R_{\mu 0}^{4}}{16 m_{e r} Z r^{8}}
$$


The calculation of the $1 / r^{n}$-perturbation for the Kepler problem can be found, e.g., in [14] (the treatment for the cases $n=2$ and $n=3$ can be found also in the textbook [15]). For the Coulomb potential $-\alpha / r$ perturbed by the potential $-\beta / r^{n+1}$, the orbit undergoes a precession with the perihelion advance:

$$
\delta \Phi=2 m \beta \frac{\partial}{\partial L}\left(\frac{1}{L} p^{1-n} \int_{0}^{\pi}\left(1+\varepsilon_{e} \cos \varphi\right)^{n-1} \mathrm{~d} \varphi\right)
$$

where $m$ is the reduced mass of the pair "particle-coulomb center", $L$ is the angular momentum of the particle, $\varepsilon_{e}$ is the eccentricity of its orbit, and $p=L^{2} /(m \alpha)$, in our case the particle being the electron. The ratio of the precession frequency to the Kepler frequency is the perihelion advance scaled by $2 \pi$, so for the second term in Equation (15) we obtain the following ratio of the precession frequency in the plane of the orbit to the Kepler frequency:

$$
\frac{\omega_{p i p}^{(1)}}{\omega_{K}}=-\frac{3}{4} m_{e r}^{2}(Z-1) S^{2}
$$

where

$$
S=\frac{R_{\mu 0}}{L^{2}}
$$

The same result could be obtained by using Equation (1.7.10) from book [16], where the potential corresponds to the gravitational potential of the oblate Earth and is mathematically equivalent to Equation (13) (without the last term) with the following correspondence of the quantities:

$$
G M m \Leftrightarrow(Z-1) e^{2}, I_{2} R^{2} \Leftrightarrow \frac{2 \gamma}{e^{2}}, p \Leftrightarrow \frac{Z-1}{2|E|}, \gamma=\frac{e^{2} R_{\mu 0}^{2}}{4(Z-1)}
$$

(the same treatment was used in paper [6]). Substituting the corresponding quantities into Equation (1.7.10) from book [16] and considering the case of $\theta=\pi / 2$, we obtain the same result as in Equation (17) (with $e=1$ in the atomic units).

As an example, Figure 1 shows the plot of the ratio $\left|\omega_{p i p}^{(1)} / \omega_{K}\right|$ versus $R_{\mu 0}$ for $L=3$ and $Z=2$. It is seen that in these ranges of $R_{\mu 0}$, the precession frequency $\omega_{\text {pip }}^{(1)}$ remains sufficiently smaller than the Kepler frequency of the electron $\omega_{K}$, which is the condition of the validity of the analytical result for $\omega_{p i p}^{(1)} / \omega_{K}$ from Equation (17).

Applying Equation (16) for the second perturbing term (the last term in Equation (15)), which corresponds to the effective potential due to the low eccentricity of the muon-nucleus orbits, we obtain the following additional contribution to the precession frequency (scaled by the Kepler frequency $\omega_{K}$ of the electron):

$$
\begin{aligned}
& \frac{\omega_{p i p}^{(2)}}{\omega_{K}}=-\frac{63 \varepsilon^{2} m_{\mu r} m_{e r}^{6}(Z-1)^{6} S^{7} f\left(E_{s}\right)}{256 Z}, \\
& f\left(E_{s}\right)=429-495 E_{s}+135 E_{s}^{2}-5 E_{s}^{3}
\end{aligned}
$$




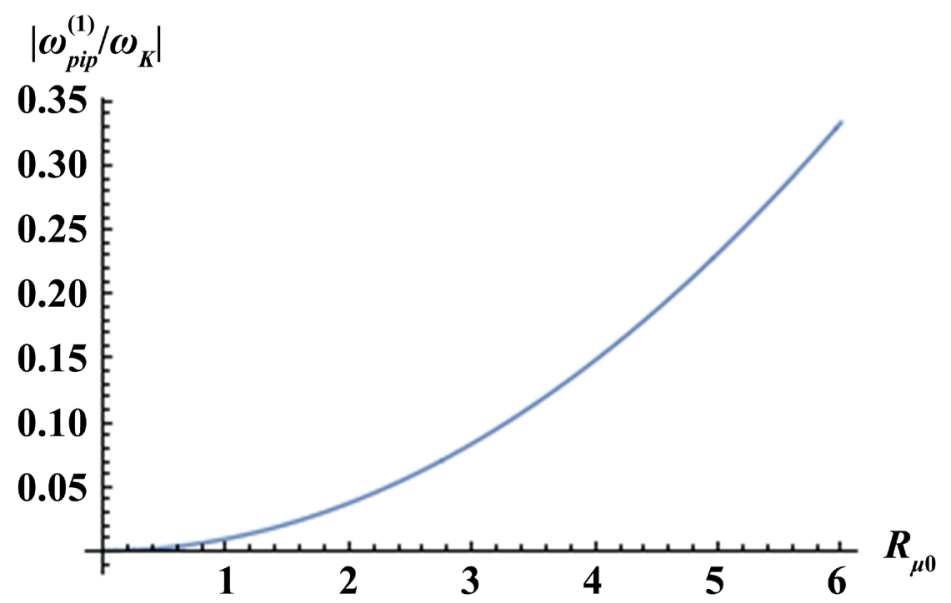

Figure 1. Plot of the ratio $\left|\omega_{\text {pip }}^{(1)} / \omega_{K}\right|$ from Equation (17) versus $R_{\mu 0}$ for $L=3$ and $Z=2$.

where

$$
E_{s}=\frac{2|E| L^{2}}{m_{e r}(Z-1)^{2}}
$$

is the absolute value of the scaled dimensionless energy of the electron. For the bounded motion of the electron, the eccentricity of its orbit is $\mathcal{E}_{e}=\left(1-E_{s}\right)^{1 / 2}$ and $0<E_{s} \leq 1$ ( $E_{s}$ is the squared ratio of the semi-minor axis to the semi-major axis of the unperturbed elliptical orbit).

Figure 2 shows the plot of the function $f\left(E_{s}\right)$ from Equation (20). As $E_{s}$ increases from 0 to $1, f\left(E_{s}\right)$ monotonically decreases from 429 to 64 .

As an example, Figure 3 shows the plot of the ratio $\left|\omega_{p i p}^{(2)} / \omega_{K}\right|$ versus $R_{\mu 0}$ and $\varepsilon$ for $E_{s} \ll 1$ (corresponding to the relatively large eccentricity of the electron orbit $\left.\left(1-\varepsilon_{e}\right) \ll 1\right), L=3$, and $Z=2$ (so that $m_{\mu r}=200.4$ ). It is seen that in these ranges of $R_{\mu 0}$ and $\varepsilon$, the additional precession frequency $\omega_{p i p}^{(2)}$ remains sufficiently smaller than the Kepler frequency of the electron $\omega_{K}$, which is the condition of the validity of the analytical result for $\omega_{\text {pip }}^{(2)} / \omega_{K}$ from Equation (20).

The ratio $\omega_{\text {pip }}^{(2)} / \omega_{\text {pip }}^{(1)}$ (denoted below as $K_{21}$ ) of the additional contribution to the precession frequency from Equation (20) to the primary contribution to the precession frequency from Equation (17) is:

$$
K_{21}=\frac{21 \varepsilon^{2} m_{\mu r} m_{e r}^{4}(Z-1)^{5} S^{5} f\left(E_{s}\right)}{64 Z}
$$

Figure 4 shows the plot of $K_{21}$ versus $S=R_{\mu 0} / L^{2}$ and $E_{s}$ (defined in Equation (21)) for $Z=2$ and $\varepsilon=0.02$.

Figure 5 presents the plot of the ratio $K_{21}$ versus $S$ and $Z$ for $\varepsilon=0.02$ and $E_{s} \ll 1$.

Figure 6 shows the plot of the ratio $K_{21}$ versus $R_{\mu 0}$ and $\varepsilon$ for $Z=2, L=3$, and $E_{s} \ll 1$.

From Figures 4-6 it is seen that within the ranges of the parameters, where the analytical results for $\omega_{p i p}^{(1)} / \omega_{K}$ from Equation (17) and for $\omega_{p i p}^{(2)} / \omega_{K}$ from 


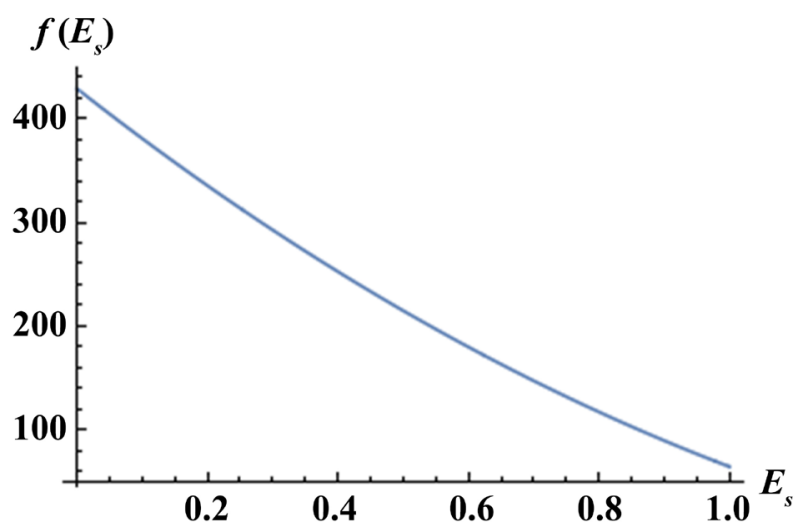

Figure 2. Plot of the function $f\left(E_{s}\right)$ from Equation (20), where $E_{s}$ is the absolute value of the scaled dimensionless energy of the electron defined in Equation (21).

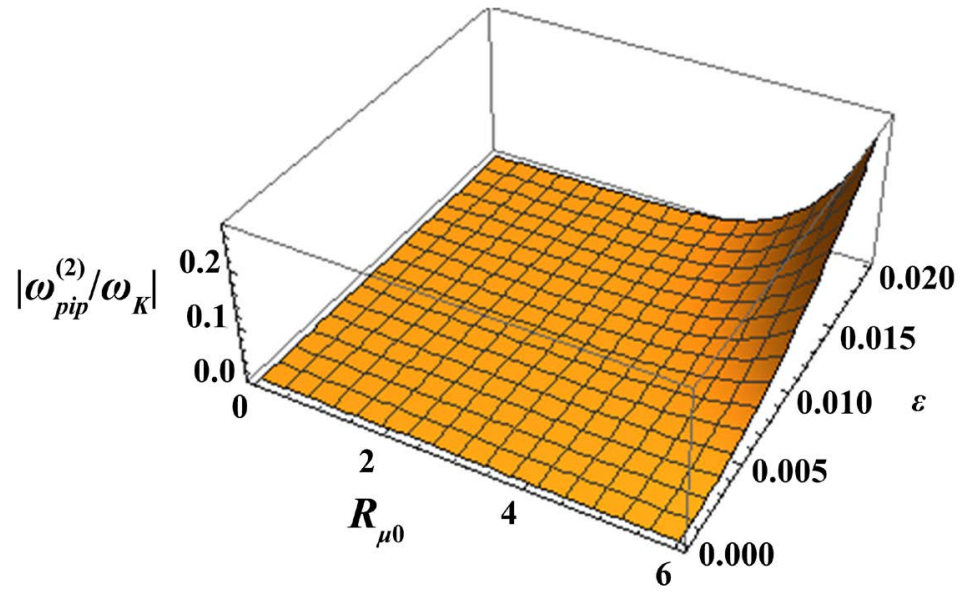

Figure 3. Plot of the ratio $\left|\omega_{p i p}^{(2)} / \omega_{K}\right|$ from Equation (20) versus $R_{\mu 0}$ and $\varepsilon$ for $E_{s} \ll 1$ (corresponding to the relatively large eccentricity of the electron orbit $\left.\left(1-\varepsilon_{e}\right) \ll 1\right), L=$ 3, and $Z=2$ (so that $m_{\mu r}=200.4$ ).

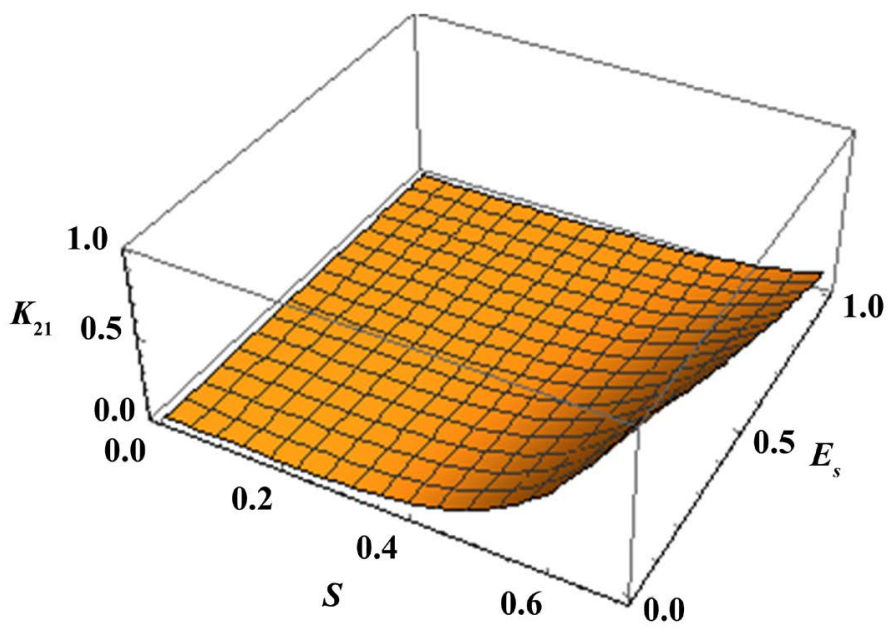

Figure 4. Plot of the ratio $K_{21}$ of the additional contribution to the precession frequency from Equation (19) to the primary contribution to the precession frequency from Equation (17) versus $S=R_{\mu 0} / L^{2}$ and $E_{s}$ (defined in Equation (21)) for $Z=2$ and $\varepsilon=0.02$. 


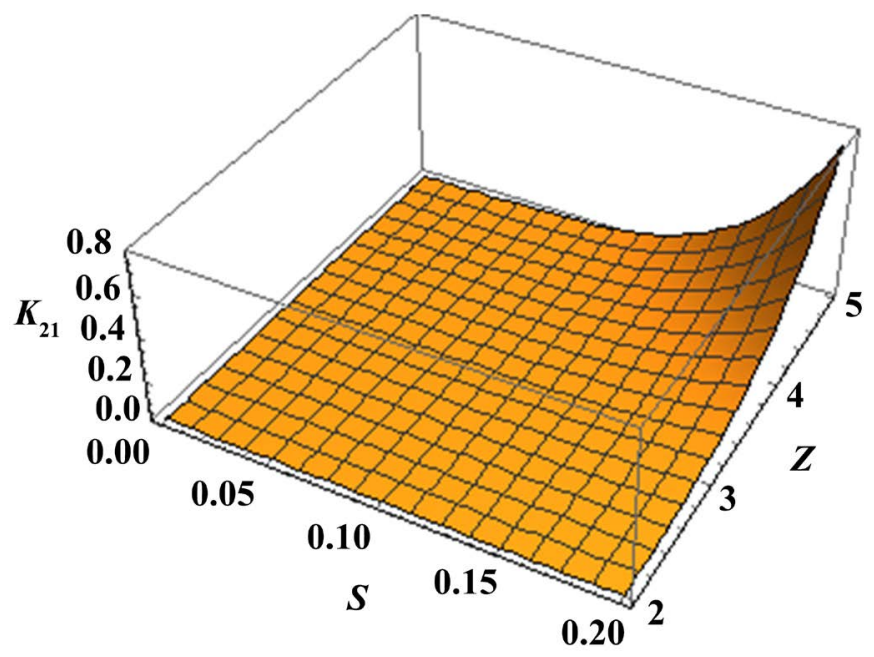

Figure 5. Plot of the ratio $K_{21}$ versus $S$ and $Z$ for $\mathcal{\varepsilon}=0.02$ and $E_{s} \ll 1 \quad\left(E_{s} \ll 1\right.$ corresponds to a relatively large eccentricity of the unperturbed electron orbit).

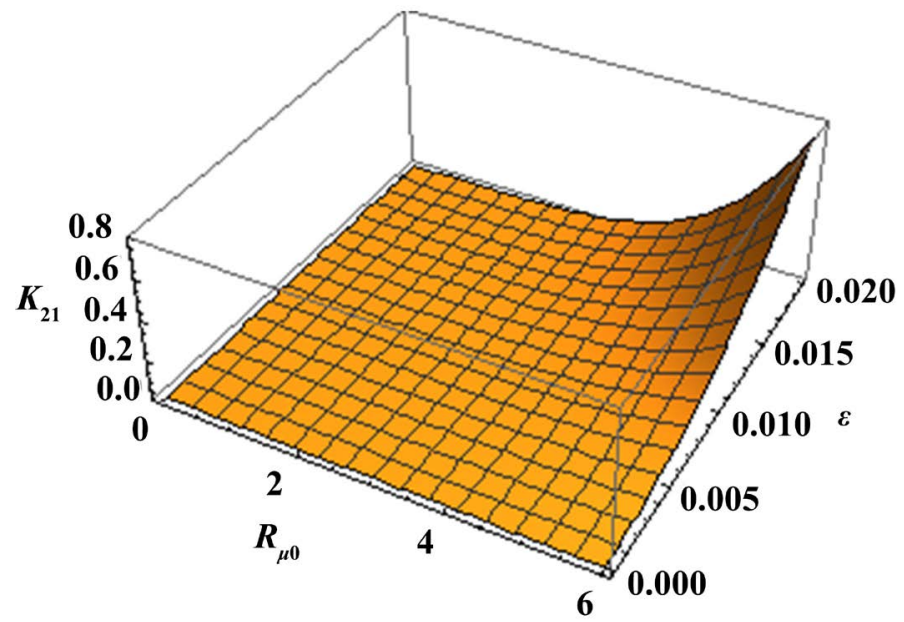

Figure 6. Plot of the ratio $K_{21}$ versus $R_{\mu 0}$ and $\varepsilon$ for $Z=2, L=3$, and $E_{s} \ll 1$.

Equation (20) remain valid, the additional contribution $\omega_{\text {pip }}^{(2)}$ precession frequency, caused by a relatively small eccentricity of the muon orbit (and of the nucleus orbit), can reach the same order of magnitude as the primary contribution $\omega_{p i p}^{(1)}$. This was the sole purpose of the above illustrations.

\section{Conclusion}

We considered a situation where the muon and nucleus orbits in the "nucleusmuon-electron" system are elliptical-the situation is more general compared to paper [6], where the muon and nucleus orbits were set to be circular. For the case where the eccentricity $\varepsilon$ of the muon and nucleus orbits is relatively small, we obtained an additional, $\varepsilon$-dependent term in the effective potential for the motion of the electron. By analytical calculations, we demonstrated that in the particular case of the planar geometry (where the electron orbit is in the plane of the muon and nucleus orbits), it leads to an additional contribution to the fre- 
quency of the precession of the electron orbit. We showed that this additional, $\varepsilon$-dependent contribution to the precession frequency of the electron orbit can reach the same order of magnitude as the primary, $\varepsilon$-independent contribution to the precession frequency. Thus, the results of our paper seem to be important not only qualitatively, but also quantitatively.

\section{Funding}

This research received no external funding.

\section{Conflicts of Interest}

The authors declare no conflict of interest.

\section{References}

[1] Chelkowsky, C., Bandrauk, A.D. and Corkum, P.B. (2004) Muonic Molecules in Superintense Laser Fields. Physical Review Letters, 93, Article No. 083602. https://doi.org/10.1103/PhysRevLett.93.083602

[2] Nagamine, K. and Ponomarev, L.I. (2003) Recent Progress in Muon Catalyzed Fusion. Nuclear Physics A, 721, C863-C866. https://doi.org/10.1016/S0375-9474(03)01227-2

[3] Guffin, J., Nixon, G., Javorsek II, D., Colafrancesco, S. and Fischbach, E. (2002) Astrophysical Detection of Heavy-Particle-Induced Spectral Shifts in Muonic Iron. Physical Review D, 66, Article No. 123508. https://doi.org/10.1103/PhysRevD.66.123508

[4] Nagamine, K. (2001) Review of Muon Catalyzed Fusion Experiments-Activities after EXAT98 and Future Perspectives. Hyperfine Interactions, 138, 5-13. https://doi.org/10.1023/A:1020822011511

[5] Ponomarev, L.I. (1990) Muon Catalysed Fusion. Contemporary Physics, 31, 219-245. https://doi.org/10.1080/00107519008222019

[6] Oks, E. (2020) Classical Analytical Solution for Rydberg States of Muonic-Electronic Helium and Helium-Like Ions. Canadian Journal of Physics, 98, 857-861. https://doi.org/10.1139/cjp-2019-0483

[7] Kryukov, N. and Oks, E. (2014) Muonic-Electronic Quasi Molecules Based on a Fully Stripped Multicharged Ion. Canadian Journal of Physics, 92, 1405-1410. https://doi.org/10.1139/cjp-2013-0705

[8] Kryukov, N. and Oks, E. (2013) Muonic-Electronic Negative Hydrogen Ion: Circular States. Canadian Journal of Physics, 91, 715-721. https://doi.org/10.1139/cjp-2013-0077

[9] Nadezhdin, B.B. and Oks, E. (1986) Highly Excited Atoms in Linearly Polarized High-Frequency Electromagnetic Radiation. Soviet Technical Physics Letters, 12, 512-513.

[10] Kapitza, P.L. (1951) Dynamic Stability of the Pendulum with Vibrating Suspension Point. Soviet Physivs JETP, 21, 588-597.

[11] Kapitza, P.L. (1951) A Pendulum with a Vibrating Suspension. Uspekhi Fizicheskikh Nauk, 44, 7-20.

[12] Nadezhdin, B.B. (1986) Radiatsionnye i Relativistskie Effekty v Atomakh i Ionakh (Radiative and Relativistic Effects in Atoms and Ions). Scientific Council of the USSR Academy of Sciences on Spectroscopy, Moscow, 222 p. (In Russian) 
[13] Oks, E. (2020) Analytical Advances in Quantum and Celestial Mechanics: Separating Rapid and Slow Subsystems. IOP Publishing, Bristol, UK.

[14] Belitz, D. (2014) Lectures on Theoretical mechanics. 95 p. https://pages.uoregon.edu/dbelitz/teaching/lecture notes/Mechanics\%20(DB)/611 2 Lecture Notes.pdf

[15] Landau, L.D. and Lifshitz, E.M. (1960) Quantum Mechanics. In: Sykes, J.B. and Bell, J.S., Eds, Course of Theoretical Physicis, Vol. 3, Problem 3, Pergamon Press, Oxford.

[16] Beletsky, V.V. (2001) Essays on the Motion of Celestial Bodies. Birkhäuser/Springer, Basel, Switzerland. https://doi.org/10.1007/978-3-0348-8360-3 